\title{
Bronchopulmonary dysplasia: a crime of opportunity?
}

\author{
Aaron C. Ericsson \\ Affiliation: University of Missouri Metagenomics Center (MUMC), Dept of Veterinary Pathobiology, University \\ of Missouri, Columbia, MO, USA. \\ Correspondence: Aaron C. Ericsson, University of Missouri, 4011 Discovery Drive, Columbia, M0 65201, USA. \\ E-mail: ericssonadmissouri.edu
}

@ERSpublications

The manuscript by Gallacher and co-workers reveals specific bacteria associated with innate immune responses in ventilated preterm infants affected with bronchopulmonary dysplasia https://bit.ly/2Xfaq $4 \mathrm{~b}$

Cite this article as: Ericsson AC. Bronchopulmonary dysplasia: a crime of opportunity? Eur Respir J 2020; 55: 2000551 [https://doi.org/10.1183/13993003.00551-2020].

First described in 1967, one of the most vexing problems in the care of preterm infants continues to be bronchopulmonary dysplasia (BPD). The clinical presentation and pathological changes associated with $\mathrm{BPD}$, also referred to as chronic lung disease of prematurity, have changed substantially since that initial description by NoRThway et al. [1]. The condition described in that seminal report, characterised by marked respiratory distress associated with pulmonary oedema due to shunting across the patent ductus arteriosus, was also specific to preterm infants that had received high inspired oxygen concentrations for at least a week. This newly recognised form of respiratory failure was attributed to aggressive mechanical ventilation and hyper-oxygenation, as it was typified by pulmonary hypertension and oedema, and cor pulmonale. Thus, those astute observations of a clinical syndrome likely occurring in hospitals across the world led to changes in the use of mechanical ventilation in preterm infants, and innovative research resulting in ground-breaking treatments such as antenatal glucocorticoids and synthetic surfactant therapy. While these approaches in the care of preterm infants dramatically improved survival rates of preterm infants and markedly reduced the incidence of the BPD reported by NorTHwAY et al. [1], BPD did not disappear - it just changed.

Clinical BPD in the post-surfactant era now primarily affects severely preterm infants, and results in very different lesions from classic BPD, suggesting arrested lung development rather than injury and repair. Moreover, while mortality associated with BPD is now very low, overall incidence has remained relatively steady for the past several decades [2], and survivors who experienced only mild respiratory distress in the perinatal period are still predisposed to late respiratory disease during childhood and adulthood, including increased risks for reactive airway disease, exercise intolerance, and other adverse sequelae [3]. Thus, there is a continued interest in the identity and influence of genetic [4] and environmental factors, such as adrenal insufficiency [5], and ante- and post-natal infections on the development of BPD.

Of particular note, three independent reports emerged in 1988 suggesting a causal relationship between Ureaplasma urealyticum in the lower airways and BPD in separate cohorts of preterm infants [6-8]. While isolates from the phylum Firmicutes such as Staphylococcus and Streptococcus spp. were also reported, U. urealyticum (phylum Tenericutes) was the most common isolate from preterm infants in all three studies and was significantly associated with the development of BPD. Moreover, many of the affected infants in these reports were delivered with intact membranes suggesting in utero exposure [6]. Ureaplasma seemed like the perfect culprit as an infectious agent driving the incidence of $\mathrm{BPD}$, as it is 
rather difficult to culture ex vivo, stains poorly with Gram stain due to its lack of a cell wall, and is thus not susceptible to certain antibiotics commonly used to treat neonatal infections [6], i.e. beta-lactams. Follow-up studies documented maternal transmission to preterm infants $[9,10]$, and pneumonic responses associated with infection [11].

Since those initial reports, however, a controversy of sorts developed regarding the influence of $U$. urealyticum in the development of BPD in preterm infants. As the first culture-independent methods of detecting specific bacteria (e.g. PCR) or profiling mixed bacterial communities (e.g. T-RFLP, DGGE) were applied to the question, associations between Ureaplasma sp. and BPD were both supported [12-14] and refuted $[15,16]$. Subsequent studies published in the past decade and employing more refined methods based on bacterial 16S rRNA gene sequences detected in endotracheal aspirates and other samples have, in general, supported an association between Ureaplasma sp. and BPD [17-20], although this finding is still not consistent across all studies [21]. Similarly, a detailed meta-analysis of the predicted effect of azithromycin and other macrolides targeting Ureaplasma sp. on the development of BPD produced mixed results, with no clear-cut protective effect from antibiotics known to act against the clinical isolates [22].

Collectively, these studies simply reinforce that BPD is a multi-factorial condition. While U. urealyticum likely contributes to harmful inflammatory responses in some situations, it is not the sole aetiological agent driving the development of BPD. Additionally, these studies have occurred as part of a larger corpus of work revealing rich bacterial communities in the lungs of healthy adults. While the lower airways and lungs were not included in phase I of the Human Microbiome Project [23] study design, based on the belief that these tissues were more or less sterile during health, it is now largely accepted that there are low biomass microbial communities constitutively present in the lungs of healthy individuals. Considering their proximity to the richly colonised oropharynx and the constant introduction of air (itself containing microbes) through the oral cavity and nares, it makes perfect sense that bacteria would reach the distal airways. It is also a testament to the mammalian immune system that the bacteria and fungi reaching our lower airways are maintained at such a low level as to render them undetectable for so many years. Indeed, earlier culture-based studies typically failed to grow the fastidious organisms detected in the lungs of healthy individuals [24], and there is very little evidence histologically of bacterial cells in the lungs. Highly sensitive, culture-independent methods have, however, demonstrated the presence of these bacterial communities in bronchoalveolar lavage fluid collected aseptically from healthy humans [25] and multiple other host species [26-28]. Notably, these lung communities appear to be better predictors of baseline innate immunity than oral or gut microbiotas in rodent models [29], and are often substantially changed in the setting of chronic respiratory disease [25, 30].

In term infants, the lung microbiota is presumably established at birth [31] via aspiration of bacteria in the oral cavity introduced from myriad environmental sources, including maternal milk microbiota [32], and repeatedly inoculated throughout life by subclinical micro-aspirations. Very little is known, however, regarding the ontogeny of the lung microbiota in preterm infants and the effects on inflammatory responses in the newborn. This is of great clinical significance, as maternal and fetal immune responses may have different prognostic implications for the preterm infant. Considering the reported influence of the lung microbiota on basal innate immunity in mice, it is important to consider candidate infectious agents in the context of the surrounding microbiota.

In this issue of European Respiratory Journal, GALLACHER et al. [33] describe the results of a longitudinal microbial survey of the lungs, upper airways, and stool of 55 ventilated, preterm infants from two recruitment sites, with a combined BPD incidence of $84 \%$. Importantly, infants were assessed longitudinally to identify patterns over time. Applying qPCR and $16 \mathrm{~S}$ rRNA-based approaches to a starting total of 1102 samples, the authors demonstrate the gradual colonisation of the upper and lower airways, punctuated by transient spikes in biomass at different sites within the first 1 to 2 weeks of life before to receding to lower baseline levels.

One of the most striking findings with regard to the detected bacterial communities was the marked dominance in the majority of samples by a single taxon. While the upper airways and lungs of full-term infants can be similarly dominated by certain taxa, they are much more likely to be colonised by relatively similar levels of multiple genera [21,34]. The data presented by GALLACHER et al. [33] can perhaps be interpreted as evidence of a more permissive microbial environment in the preterm lung, wherein the first colonisers proliferate and gain a dominant position, as opposed to the term lung wherein host immune defences prevent over-proliferation of any given taxa and maintain a more balanced microbial distribution.

The authors also noted that those samples that failed to amplify and sequence adequately (a common occurrence with low biomass samples) were frequently those samples collected during prophylactic treatment with benzylpenicillin and gentamicin. Importantly, samples from children receiving antibiotics that did sequence well demonstrated no decrease in $\alpha$-diversity, suggesting that treatment was broadly 


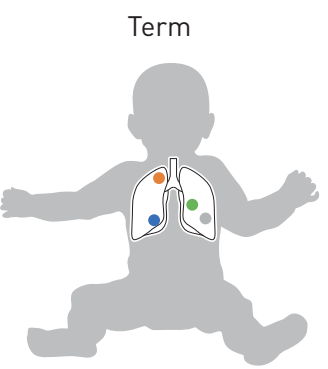

High diversity lung microbiome

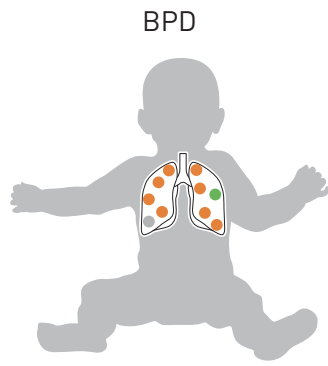

Low diversity lung microbiome

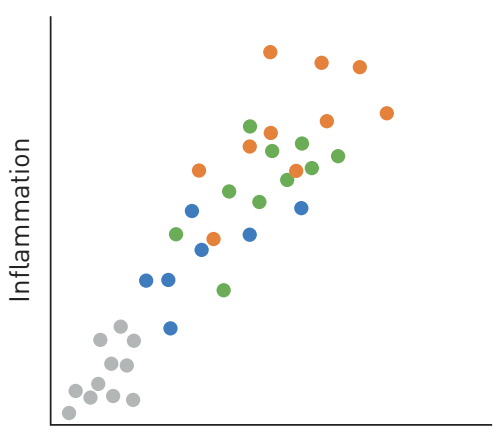

Bacterial biomass

FIGURE 1 Infants with bronchopulmonary dysplasia (BPD) are typically born pre-term, and often require mechanical ventilation.

suppressing bacterial growth, rather than selectively killing certain taxa. With that in mind, the acute phase protein IL-6 and neutrophil chemo-attractant IL- 8 were measured in each sample, and comparisons were made between samples that did or did not sequence well. While those cytokines were below the limit of detection in most fecal samples and nasopharyngeal aspirates, they were readily detectable in the tracheal aspirates and bronchoalveolar lavage fluid of preterm infants, and were significantly greater in infants providing samples that sequenced well, suggesting a link between greater levels of microbial colonisation and inflammatory responses. Focusing on that relationship over time in individual infants, there was a clear temporal relationship between microbial growth and airway inflammation, with antibiotic administration apparently mitigating both. Interestingly, the authors note that the strongest inflammatory responses were associated with specific taxa including Acinetobacter, unclassified genera within the family Enterobacteriaceae, and Mollicutes such as Mycoplasma and Ureaplasma. With that in mind, it should be noted that those latter taxa represented the dominant coloniser in a relatively small number of samples, again reinforcing the multifactorial nature of disease development.

Collectively, these data indicate that the preterm lung microbiota differs from upper airway communities, is particularly susceptible to colonisation and overgrowth by a single genus, and is significantly influenced by the dominant genus colonising the airways. Perhaps colonisation by any bacteria beyond a certain threshold invokes an inflammatory response, making BPD purely a disease of opportunity, and highlighting the limitations of Koch's postulates in the era of molecular medicine. Alternatively, perhaps select bacteria can actually provide a protective effect against opportunist agents, akin to colonisation resistance in the gut. As with those initial associations between $U$. urealyticum and BPD, the answers to good research always bring additional questions.

Conflict of interest: None declared.

\section{References}

1 Northway WH Jr, Rosan RC, Porter DY. Pulmonary disease following respirator therapy of hyaline-membrane disease. Bronchopulmonary dysplasia. N Engl J Med 1967; 276: 357-368.

2 Stoll BJ, Hansen NI, Bell EF, et al. Trends in care practices, morbidity, and mortality of extremely preterm neonates, 1993-2012. JAMA 2015; 314: 1039-1051.

3 Gough A, Spence D, Linden M, et al. General and respiratory health outcomes in adult survivors of bronchopulmonary dysplasia: a systematic review. Chest 2012; 141: 1554-1567.

$4 \mathrm{Yu} \mathrm{KH,} \mathrm{Li} \mathrm{J,} \mathrm{Snyder} \mathrm{M,} \mathrm{et} \mathrm{al.} \mathrm{The} \mathrm{genetic} \mathrm{predisposition} \mathrm{to} \mathrm{bronchopulmonary} \mathrm{dysplasia.} \mathrm{Curr} \mathrm{Opin} \mathrm{Pediatr} \mathrm{2016;}$ 28: 318-323.

5 Watterberg KL, Gerdes JS, Cole CH, et al. Prophylaxis of early adrenal insufficiency to prevent bronchopulmonary dysplasia: a multicenter trial. Pediatrics 2004; 114: 1649-1657.

6 Cassell GH, Waites KB, Crouse DT, et al. Association of Ureaplasma urealyticum infection of the lower respiratory tract with chronic lung disease and death in very-low-birth-weight infants. Lancet 1988; 2: 240-245.

7 Sanchez PJ, Regan JA. Ureaplasma urealyticum colonization and chronic lung disease in low birth weight infants. Pediatr Infect Dis J 1988; 7: 542-546.

8 Wang EE, Frayha H, Watts J, et al. Role of Ureaplasma urealyticum and other pathogens in the development of chronic lung disease of prematurity. Pediatr Infect Dis J 1988; 7: 547-551.

9 Sanchez PJ, Regan JA. Vertical transmission of Ureaplasma urealyticum from mothers to preterm infants. Pediatr Infect Dis J 1990; 9: 398-401.

10 Alfa MJ, Embree JE, Degagne P, et al. Transmission of Ureaplasma urealyticum from mothers to full and preterm infants. Pediatr Infect Dis J 1995; 14: 341-345.

11 Panero A, Pacifico L, Rossi N, et al. Ureaplasma urealyticum as a cause of pneumonia in preterm infants: analysis of the white cell response. Arch Dis Child Fetal Neonatal Ed 1995; 73: F37-F40.

12 Kotecha S, Hodge R, Schaber JA, et al. Pulmonary Ureaplasma urealyticum is associated with the development of acute lung inflammation and chronic lung disease in preterm infants. Pediatr Res 2004; 55: 61-68. 
13 Payne MS, Goss KC, Connett GJ, et al. Molecular microbiological characterization of preterm neonates at risk of bronchopulmonary dysplasia. Pediatr Res 2010; 67: 412-418.

14 Kasper DC, Mechtler TP, Bohm J, et al. In utero exposure to Ureaplasma spp. is associated with increased rate of bronchopulmonary dysplasia and intraventricular hemorrhage in preterm infants. J Perinat Med 2011; 39: 331-336.

15 Stressmann FA, Connett GJ, Goss K, et al. The use of culture-independent tools to characterize bacteria in endo-tracheal aspirates from pre-term infants at risk of bronchopulmonary dysplasia. J Perinat Med 2010; 38: 333-337.

16 Katz B, Patel P, Duffy L, et al. Characterization of ureaplasmas isolated from preterm infants with and without bronchopulmonary dysplasia. J Clin Microbiol 2005; 43: 4852-4854.

17 Beeton ML, Maxwell NC, Davies PL, et al. Role of pulmonary infection in the development of chronic lung disease of prematurity. Eur Respir J 2011; 37: 1424-1430.

18 Mourani PM, Harris JK, Sontag MK, et al. Molecular identification of bacteria in tracheal aspirate fluid from mechanically ventilated preterm infants. PLoS One 2011; 6: e25959.

19 Wagner BD, Sontag MK, Harris JK, et al. Airway microbial community turnover differs by BPD severity in ventilated preterm infants. PLoS One 2017; 12: e0170120.

20 Lohmann P, Luna RA, Hollister EB, et al. The airway microbiome of intubated premature infants: characteristics and changes that predict the development of bronchopulmonary dysplasia. Pediatr Res 2014; 76: 294-301.

21 Lal CV, Travers C, Aghai ZH, et al. The airway microbiome at birth. Sci Rep 2016; 6: 31023.

22 Nair V, Loganathan P, Soraisham AS. Azithromycin and other macrolides for prevention of bronchopulmonary dysplasia: a systematic review and meta-analysis. Neonatology 2014; 106: 337-347.

23 Human Microbiome Project C. Structure, function and diversity of the healthy human microbiome. Nature 2012; 486: $207-214$.

24 Cabello H, Torres A, Celis R, et al. Bacterial colonisation of distal airways in healthy subjects and chronic lung disease: a bronchoscopic study. Eur Respir J 1997; 10: 1137-1144.

25 Erb-Downward JR, Thompson DL, Han MK, et al. Analysis of the lung microbiome in the "healthy" smoker and in COPD. PLoS One 2011; 6: el6384.

26 Ericsson AC, Personett AR, Grobman ME, et al. Composition and predicted metabolic capacity of upper and lower airway microbiota of healthy dogs in relation to the fecal microbiota. PLoS One 2016; 11: e0154646.

27 Glendinning L, Wright S, Pollock J, et al. Variability of the sheep lung microbiota. Appl Environ Microbiol 2016; 82: $3225-3238$

28 Vientos-Plotts AI, Ericsson AC, Rindt H, et al. Dynamic changes of the respiratory microbiota and its relationship to fecal and blood microbiota in healthy young cats. PLoS One 2017; 12: e0173818.

29 Dickson RP, Erb-Downward JR, Falkowski NR, et al. The lung microbiota of healthy mice are highly variable, cluster by environment, and reflect variation in baseline lung innate immunity. Am J Respir Crit Care Med 2018; 198: 497-508.

30 Mayhew D, Devos N, Lambert C, et al. Longitudinal profiling of the lung microbiome in the AERIS study demonstrates repeatability of bacterial and eosinophilic COPD exacerbations. Thorax 2018; 73: 422-430.

31 Gallacher DJ, Kotecha S. Respiratory microbiome of new-born infants. Front Pediatr 2016; 4: 10.

32 Solis G, de Los Reyes-Gavilan CG, Fernandez N, et al. Establishment and development of lactic acid bacteria and bifidobacteria microbiota in breast-milk and the infant gut. Anaerobe 2010; 16: 307-310.

33 Gallacher D, Mitchell E, Alber D, et al. Dissimilarity of the gut-lung axis and dysbiosis of the lower airways in ventilated preterm infants. Eur Respir J 2020; 55: 1901909.

34 Biesbroek G, Tsivtsivadze E, Sanders EA, et al. Early respiratory microbiota composition determines bacterial succession patterns and respiratory health in children. Am J Respir Crit Care Med 2014; 190: 1283-1292. 